\title{
Evolution of harmonic maps on manifolds flat at infinity
}

\author{
Irene Paniccia
}

\begin{abstract}
The aim of this article is to prove a global existence result with small data for the heat flow for harmonic maps from a manifold flat at infinity into a compact manifold. By flat at infinity we mean that the growth rate of the volumes of the balls on the manifold is the same as in the flat space. This is true for any manifold for small enough radius, but is in general not true when the radius of the ball grows. So prescribing such a growth rate also at infinity selects a class of manifolds on which our result holds. In this setting estimates are available for the heat kernel and its gradient on the base manifold. From such estimates it is easy to get $L^{p}-L^{q}$ bounds for the heat kernel. A contraction principle argument then yields a local existence result in a suitable Sobolev space and a global existence result for small data.
\end{abstract}

Mathematics Subject Classification (2000). 58E20 (58J35).

Keywords. Harmonic maps, Heat flow, Homotopy problem, Heat kernel, Faber-Krahn inequality.

\section{Introduction}

Let $(\mathcal{M}, g),(\mathcal{N}, h)$ be two Riemannian manifolds of dimension $m$ and $n$, respectively. Assume $\mathcal{N}$ to be compact and without border, so that, by Nash's theorem [26], we may assume it to be isometrically embedded in $\mathbb{R}^{N}$ for some $N \geq n$. As usual, we define the energy density of a map $u=\left(u^{1}, \ldots, u^{N}\right) \in$ $C^{1}(\mathcal{M}, \mathcal{N})$ at a point $P \in \mathcal{M}$ as

$$
[e(u)](P):=\sum_{j=1}^{N}\left|\nabla u^{j}\right|^{2},
$$

where $\nabla u$ is the usual Riemannian gradient of $u$ on $\mathcal{M}$. The energy functional is defined for $u$ in $H^{1}(\mathcal{M}, \mathcal{N})$ by the integral 


$$
\begin{aligned}
E(u) & :=\frac{1}{2} \int_{\mathcal{M}} e(u) d \mu \\
& =\frac{1}{2} \int_{\mathcal{M}} \sum_{i, j=1}^{N} g^{i j} \partial_{x^{i}} u \cdot \partial_{x^{j}} u d \mu
\end{aligned}
$$

where $d \mu$ is the volume element on $(\mathcal{M}, g)$, and the critical points of $E$ are called harmonic mappings.

An important question is whether every homotopy class of maps from $\mathcal{M}$ to $\mathcal{N}$ contains a harmonic map. This is called the homotopy problem. In general, variational methods are not appropriate for this kind of problem since homotopy classes may be not weakly closed in the Sobolev space $H^{1}$.

Eells and Sampson [15] proposed to study the problem by an evolutionary approach, by constructing a harmonic map homotopic to $u_{0}$ as the asymptotic limit of a solution to the Cauchy problem

$$
\begin{cases}\partial_{t} u-\Delta u=A(u)(\nabla u, \nabla u) & \text { on } \mathcal{M} \times[0,+\infty[ \\ u=u_{0} & \text { for } t=0 \text { and on } \partial \mathcal{M} \times[0,+\infty[.\end{cases}
$$

Using this approach they solved the homotopy problem in the case when the target manifold has non-positive sectional curvature.

When the target manifold has positive sectional curvature, in general the Cauchy problem (3) does not have a global solution: we mention in particular the counterexamples to existence and uniqueness for $\mathcal{N}=\mathbb{S}^{2}$ exhibited by Chang, Ding and Ye [4] and Coron [7].

In 1990 Soyeur considered the situation when $\mathcal{M}$ is the euclidean space and $\mathcal{N}$ is an arbitrary manifold, and solved (3) globally for small data. The main advantage of this setting is that global $L^{p}-L^{q}$ estimates for the heat kernel on $\mathbb{R}^{m}$ are available. Here we shall extend Soyeur's setting and allow the base space $\mathcal{M}$ to be a more general manifold, asymptotically close in a suitable sense to $\mathbb{R}^{m}$.

We recall briefly the result of Soyeur. Let $p>m$. Consider the space

$$
\mathcal{F}_{p}:=\left\{v: \mathcal{M} \rightarrow \mathbb{R}^{N}:\|v\|_{L^{\infty}}<+\infty,\|\nabla v\|_{L^{p}}<+\infty\right\}
$$

with the norm

$$
\|v\|_{\mathcal{F}_{p}}:=\|v\|_{L^{\infty}}+\|\nabla v\|_{L^{p}} .
$$

Given $u_{0} \in L^{\infty}\left(\mathcal{M}, \mathbb{R}^{N}\right)$ with $\nabla u_{0} \in L^{p}\left(\mathcal{M}, \mathbb{R}^{m N}\right)$, let

$$
M:=2\left\|u_{0}\right\|_{\mathcal{F}_{p}} \equiv 2\left(\left\|u_{0}\right\|_{L^{\infty}}+\left\|\nabla u_{0}\right\|_{L^{p}}\right)
$$

and

$$
\mathcal{E}_{p}^{T}:=\left\{u \in C\left([0, T], \mathcal{F}_{p}\right): \sup _{t \in[0, T]}\left\|u(t)-e^{\Delta t} u_{0}\right\|_{\mathcal{F}_{p}} \leq M\right\} .
$$

with the norm $\|u\|_{\mathcal{E}_{p}^{T}}:=\sup _{t \in[0, T]}\|u(t)\|_{\mathcal{F}_{p}}$; notice that $\mathrm{E}$ thus defined is a Banach space. The main result of Soyeur is the following:

Theorem 1.1 (Soyeur [29]). Let $\mathcal{M}=\mathbb{R}^{m}$. Let $u_{0} \in \mathcal{F}_{p}, p>m$. There exist $T\left(u_{0}\right)>0$ and $u \in \mathcal{E}_{p}^{T\left(u_{0}\right)}$ smooth on $] 0, T\left(u_{0}\right)[\times \mathcal{M}$ which solves (3). Let $\bar{T}\left(u_{0}\right)$ be the maximal existence time of $u$. Then 
(i) $u \in C\left(\left[0, \bar{T}\left(u_{0}\right)\left[, \mathcal{F}_{p}\right)\right.\right.$. Moreover, if $\bar{T}\left(u_{0}\right)<+\infty$, then $\|u(t)\|_{L^{\infty}}+$ $\|\nabla u(t)\|_{L^{p}} \rightarrow+\infty$ as $t \rightarrow \bar{T}\left(u_{0}\right)$.

(ii) There exists a constant $\epsilon>0$ depending only on $\mathcal{M}$ and $\mathcal{N}$ such that if $\left\|\nabla u_{0}\right\|_{L^{m}}<\epsilon$, then $\bar{T}\left(u_{0}\right)=+\infty$. Moreover, the solution converges to a constant map as $t \rightarrow+\infty$ with the following decay:

$$
\|\nabla u(t)\|_{L^{p}} \leq \frac{M_{p}^{\infty}}{t^{\frac{1}{2}-\frac{m}{2 p}}},
$$

where the constant $M_{p}^{\infty}$ depends only on $\mathcal{M}, \mathcal{N}$ and $p$.

Remark. Soyeur also treats the case when $\mathcal{M}$ is a compact manifold and the map $u$ satisfies Dirichlet boundary conditions: $\left.u\right|_{\partial \mathcal{M}}=0 \in \mathcal{N} \subset \mathbb{R}^{N}$. In this case, a map satisfying the condition of the point (ii) converges to 0 as $t \rightarrow+\infty$, and the following decay holds for small amplitude solutions:

$$
\|\nabla u(t)\|_{L^{p}} \leq \frac{e^{-\lambda t}}{\chi(t)^{\frac{1}{2}-\frac{m}{2 p}}} M_{p}^{\infty}
$$

where $\lambda>0$ and $M_{p}^{\infty}$ depends only on $\mathcal{M}, \mathcal{N}$ and $p$, and $\chi(t)=\min (1, t)$.

Our goal here is to extend the above result to more general manifolds $\mathcal{M}$. We shall state the assumptions on $\mathcal{M}$ in a very general form, expressed in terms of the heat kernel $p_{t}(x, y)$ associated to the Laplace-Beltrami operator $\Delta$ on $\mathcal{M}$, and the volume $V(x, t)$ of the ball $B(x, t)$ of $\mathcal{M}$. We shall also require that $\mathcal{M}$ satisfies a Faber-Krahn inequality, i.e., there exists a function $\Lambda(s)$ such that, for any bounded open set $\Omega \subset \mathcal{M}$, we have

$$
\lambda_{1}(\Omega) \geq \Lambda(\mu(\Omega)),
$$

where $\lambda_{1}(\Omega)$ is the first Dirichlet eigenvalue on $\Omega$ while $\mu$ is the volume of $\Omega$.

Our first result concerns the $L^{p}-L^{q}$ decay properties of the heat flow $e^{\Delta t} u_{0}$ and its gradient on $\mathcal{M}$ :

Theorem 1.2. Let $\mathcal{M}$ be a Riemannian manifold.

(i) Let $D>2$ and $g: \mathbb{R}_{+} \rightarrow \mathbb{R}_{+}$with the doubling property $g(2 t) \leq C g(t)$. Assume that the heat kernel on $\mathcal{M}$ satisfies the estimate

$$
p_{t}(x, y) \leq \frac{1}{g(t)} \exp \left(-\frac{d^{2}(x, y)}{2 D t}\right), \quad x, y \in \mathcal{M}, \quad t>0 .
$$

Then the heat flow on $\mathcal{M}$ satisfies the decay estimate

$$
\left\|e^{\Delta t} u_{0}\right\|_{L^{p}} \leq g(t)^{-\left(\frac{1}{q}-\frac{1}{p}\right)}\left\|u_{0}\right\|_{L^{q}}, \quad 1 \leq q \leq p \leq+\infty .
$$

Moreover, if $V(x, \sqrt{t}) \sim g(t)$ and the gradient of the heat kernel satisfies

$$
\left|\nabla_{x} p_{t}(x, y)\right| \leq \frac{1}{t^{\frac{1}{2}}} \frac{1}{g(t)} \exp \left(-\frac{d^{2}(x, y)}{2 D t}\right), \quad x, y \in \mathcal{M}, \quad t>0
$$

we have in addition

$$
\left\|\nabla e^{\Delta t} u_{0}\right\|_{L^{p}} \leq \frac{C}{t^{\frac{1}{2}}} g(t)^{-\left(\frac{1}{q}-\frac{1}{p}\right)}\left\|u_{0}\right\|_{L^{q}}, \quad 1 \leq q \leq p \leq+\infty .
$$


(ii) Assume $\mathcal{M}$ has euclidean volume growth $V(x, \sqrt{t}) \sim t^{m / 2}$, that it satisfies a Faber-Krahn inequality with $\Lambda(s)=c s^{-\frac{2}{m}}$, and that for some $C>0$

$$
\left|\nabla_{x} p_{t}(x, y)\right| \leq C\left|\nabla_{y} p_{t}(x, y)\right|, \quad \forall x, y \in \mathcal{M}
$$

Then the heat flow satisfies

$$
\left\|e^{\Delta t} u_{0}\right\|_{L^{p}} \leq C \frac{\left\|u_{0}\right\|_{L^{q}}}{t^{\frac{m}{2}\left(\frac{1}{q}-\frac{1}{p}\right)}}, \quad 1 \leq q \leq p \leq+\infty .
$$

and

$$
\left\|\nabla e^{\Delta t} u_{0}\right\|_{L^{p}} \leq C \frac{\left\|u_{0}\right\|_{L^{q}}}{t^{\frac{1}{2}+\frac{m}{2}\left(\frac{1}{q}-\frac{1}{p}\right)}}, \quad 1 \leq q \leq p \leq+\infty .
$$

The estimates (9-10) also hold when $\mathcal{M}$ is a Galois covering of a compact manifold, with nilpotent deck transformation group $G$.

In our second result we extend Soyeur's result to the more general case of a base manifold $\mathcal{M}$ satisfying the above assumptions. Under these assumptions on $\mathcal{M}$ we obtain global well posedness for small data in the Sobolev class $\mathcal{F}_{p}$ :

Theorem 1.3. Let $\mathcal{M}$ be a manifold of dimension $m \geq 2$ such that estimates (9-10) hold, and let $\mathcal{N}$ be a compact manifold. Then for any $u_{0} \in \mathcal{F}_{p}$ with $p>m$ there exist $T\left(u_{0}\right)>0$ and $u \in \mathcal{E}_{p}^{T\left(u_{0}\right)}$ smooth on $] 0, T\left(u_{0}\right)[\times \mathcal{M}$ solution of the Cauchy problem (3). Denoting by $\bar{T}\left(u_{0}\right)$ the maximal existence time of $u$, we have in addition:

(i) $u \in C\left(\left[0, \bar{T}\left(u_{0}\right)\left[, \mathcal{F}_{p}\right)\right.\right.$, and if $\bar{T}\left(u_{0}\right)<+\infty$, then $\|u(t)\|_{L^{\infty}}+\|\nabla u(t)\|_{L^{p}} \rightarrow$ $+\infty$ as $t \rightarrow \bar{T}\left(u_{0}\right)$.

(ii) There exists a constant $\epsilon>0$ depending only on $\mathcal{M}$ and $\mathcal{N}$ such that $\bar{T}\left(u_{0}\right)=+\infty$ for $\left\|\nabla u_{0}\right\|_{L^{m}}<\epsilon$. The global solution converges to a constant map as $t \rightarrow+\infty$ with the following decay:

$$
\|\nabla u(t)\|_{L^{p}} \leq \frac{M_{p}^{\infty}}{t^{\frac{1}{2}-\frac{m}{2 p}}},
$$

where $M_{p}^{\infty}$ only depends on $\mathcal{M}, \mathcal{N}$ and $p$.

Notice that the last estimate does not express any decay when $p \leq m$, and indeed the a priori estimates in the proof of Theorem 1.3 depend in an essential way on the condition $p>m$.

Remark. The compactness assumption on $\mathcal{N}$ is used here only to get the following bound on the second fundamental form $A$ of $\mathcal{N}$ and its first derivatives:

$$
|A(u)(\nabla u, \nabla u)|+\left|A^{\prime}(u)(\nabla u, \nabla u)\right| \leq C|\nabla u|^{2} .
$$

Estimate (11) is trivially true when $\mathcal{N}$ is compact, but Theorem 1.3 holds more generally for any manifold $\mathcal{N}$ satisfying (11). 


\section{Geometric background}

In this section we are going to deal with heat kernel estimates, in particular we will give sufficient conditions on the manifold $\mathcal{M}$ to guarantee the validity of Theorem 1.2 and, in consequence of that, of Theorem 1.3. Our aim is to give nontrivial examples of manifolds satisfying such estimates. The estimates of Theorem 1.2 follow from some well-known heat kernel estimates which in turn have a deep correlation with the geometry of the manifold $\mathcal{M}$. In this section we recall briefly the standard theory of such estimates and show how to deduce Theorem 1.2 from them. For an excellent account of the theory, and detailed proofs, see e.g. the works of Grigor'yan [18, 20,22,23].

It is well known (see e.g. [5]) that the heat semigroup $e^{\Delta t}$ possesses a unique integral kernel $p_{t}(x, y)$, which is a $C^{\infty}$ function on $\mathbb{R}_{+} \times \mathcal{M} \times \mathcal{M}$. Such integral kernel is a fundamental solution to the heat equation, i.e.

$$
\begin{gathered}
\partial_{t} p_{t}(x, y)-\Delta p_{t}(x, y)=0 \quad \forall y \in \mathcal{M}, \\
p_{t}(\cdot, y) \rightarrow \delta_{y} \quad \text { as } t \rightarrow 0^{+} \quad \forall y \in \mathcal{M}, \\
p_{t}(x, y) \geq 0 \quad \forall x, y \in \mathcal{M}, \quad \forall t \in \mathbb{R}_{+}
\end{gathered}
$$

and it is regular in the sense that it satisfies the maximum principle:

$$
\int_{\mathcal{M}} p_{t}(x, y) d \mu(x) \leq 1 \quad \forall y \in \mathcal{M}, \forall t \in \mathbb{R}_{+} .
$$

Furthermore, $p_{t}(x, y)$ satisfies the following additional properties:

- Symmetry:

$$
p_{t}(x, y)=p_{t}(y, x)
$$

- The semigroup identity:

$$
p_{t+s}(x, y)=\int_{\mathcal{M}} p_{t}(x, z) p_{s}(z, y) d \mu(z) .
$$

Definition. The unique smooth integral kernel for the heat semigroup $e^{\Delta t}$ is also called the heat kernel of the manifold $(\mathcal{M}, g)$.

From now on the symbol $e^{\Delta t}$ will be used to denote the integral operator with kernel $p_{t}(x, y)$.

A trivial consequence of the properties of the heat kernel is that $p_{2 t}(x, x)=\left\|p_{t}(x, \cdot)\right\|_{L^{2}}^{2}$, which in particular implies that $p_{t}(x, \cdot)$ is in $L^{2}$.

\subsection{Kernel estimates}

Our first goal will be to find sufficient conditions for the following Gaussian estimate of the heat kernel to hold:

$$
p_{t}(x, y) \leq \frac{1}{g(t)} \exp \left(-\frac{d^{2}(x, y)}{2 D t}\right), \quad x, y \in \mathcal{M}, t>0 .
$$

In a moment we will see that on-diagonal estimates imply off-diagonal ones, so we only need to prove the bound

$$
p_{t}(x, x) \leq \frac{1}{g(t)}, \quad x \in \mathcal{M}, \quad t>0 .
$$


The last part of the section will be dedicated to geometrical conditions on the manifold $\mathcal{M}$ under which such a bound holds with $g(t)=C t^{\frac{m}{2}}$.

Let $\mathcal{M}$ be an arbitrary Riemannian manifold. Consider the following weighted integral of the heat kernel:

$$
E_{D}(x, t):=\int_{\mathcal{M}} p_{t}(x, z)^{2} \exp \left(\frac{d^{2}(x, z)}{D t}\right) d \mu(z),
$$

with $D>0$ to be specified.

Lemma 2.1 ([19]). Let $D>0$. For every $x, y \in \mathcal{M}, t>0$ it holds

$$
p_{t}(x, y) \leq \sqrt{E_{D}\left(x, \frac{t}{2}\right) E_{D}\left(y, \frac{t}{2}\right)} \exp \left(-\frac{d^{2}(x, y)}{2 D t}\right) .
$$

A priori it is not clear that $E_{D}(x, t)$ is finite. Indeed, it is easy to see that for $\mathcal{M}=\mathbb{R}^{m}, E_{D}=\infty$ for every $D \leq 2$. However, we have the following

Theorem 2.2 ([17,19]). For a manifold $\mathcal{M}, E_{D}(x, t)$ is finite for every $D>$ $2, t>0, x \in \mathcal{M}$. Moreover, $E_{D}(x, t)$ in non-increasing in $t$.

If we have on-diagonal estimates, we can easily get off-diagonal ones:

Theorem 2.3 ([33,21]). Assume that for some $x \in \mathcal{M}$ and for every $t>0$

$$
p_{t}(x, x) \leq \frac{C}{f(t)},
$$

where $f(t)$ is a positive increasing function on ]0, $+\infty[$ satisfying the following regularity condition: there exist $A \geq 1$ and $a>1$ such that

$$
\frac{f(a s)}{f(s)} \leq A \frac{f(a t)}{f(t)}, \quad \forall 0<s<t .
$$

Then, for every $D>2$ and $t>0$,

$$
E_{D}(x, t) \leq \frac{C^{\prime}}{f(\epsilon t)}
$$

for some $\epsilon>0$ and $C^{\prime}$.

Summing up, we see that a diagonal assumption like (20) is enough to prove the uniform Gaussian estimate (9) of Theorem 1.2. A large class of examples is given by manifolds satisfying a Faber-Krahn inequality:

Definition. Let $\Lambda$ be a function on $] 0,+\infty[$. We say that a Riemannian manifold $\mathcal{M}$ satisfies the (uniform) Faber-Krahn inequality with function $\Lambda$ if, for any non-empty precompact open set $\Omega \subset \mathcal{M}$,

$$
\lambda_{1}(\Omega) \geq \Lambda(\mu(\Omega)),
$$

where $\lambda_{1}(\Omega)$ is the first eigenvalue of the Laplace operator on $\Omega$ with Dirichlet boundary conditions:

$$
\lambda_{1}(\Omega):=\inf \left\{\frac{\int_{\Omega}|\nabla f|^{2}}{\int_{\Omega} f^{2}}, f \in C_{c}^{\infty}(\Omega)\right\} .
$$


The Faber-Krahn condition is a deep property of the manifold, which is equivalent to several apparently unrelated properties, and in particular to the diagonal kernel estimate we need.

Theorem 2.4. The condition

$$
\sup _{x \in \mathcal{M}} p_{t}(x, x) \leq C t^{-\frac{m}{2}}, \quad \forall t>0,
$$

is equivalent to each of the following (all true for any non-negative function $f \in \mathscr{D})$ :

(i) The Sobolev inequality:

$$
\left(\int_{\mathcal{M}} f^{\frac{2 m}{m-2}} d \mu\right)^{\frac{m-2}{m}} \leq C \int_{\mathcal{M}}|\nabla f|^{2} d \mu
$$

provided $m>2$;

(ii) The Nash inequality:

$$
\left(\int_{\mathcal{M}} f d \mu\right)^{-\frac{4}{m}}\left(\int_{\mathcal{M}} f^{2} d \mu\right)^{1+\frac{2}{m}} \leq C \int_{\mathcal{M}}|\nabla f|^{2} d \mu
$$

(iii) The one-parameter log-Sobolev inequality: for any $\epsilon>0$

$$
\int_{\mathcal{M}} f^{2} \log \left(\frac{f}{\|f\|_{L^{2}}}\right) d \mu \leq \epsilon \int_{\mathcal{M}}|\nabla f|^{2} d \mu+\left(C-\frac{m}{4} \log \epsilon\right) \int_{\mathcal{M}} f^{2} d \mu
$$

(iv) The Faber-Krahn inequality: for all non-empty precompact open sets $\Omega \subset \mathcal{M}$,

$$
\lambda_{1}(\Omega) \geq C \mu(\Omega)^{-\frac{2}{m}} .
$$

The proof of this result is spread in a number of papers: see for example $[34,2,8,10-12,3,19]$.

If we don't require the equivalence, we have a more general result for generic Faber-Krahn function $\Lambda$ :

Theorem 2.5 ([19]). Assume that the manifold $\mathcal{M}$ admits a Faber-Krahn function $\Lambda$. Let us define the function $f(t)$ by

$$
t=\int_{0}^{f(t)} \frac{d v}{v \Lambda(v)}
$$

assuming the convergence of the integral at 0 . Then for all $t>0, x \in \mathcal{M}$, and $\epsilon>0$,

$$
p_{t}(x, x) \leq \frac{2 \epsilon^{-1}}{f((1-\epsilon) t)} .
$$

Summing up, we have showed that if $\mathcal{M}$ satisfies the Faber-Krahn inequality with $\Lambda(s)=s^{-\frac{2}{m}}$, then the Gaussian kernel estimate of Theorem 1.2 holds. 


\subsection{Gradient estimates}

The second ingredient in Theorem 1.2 is a Gaussian estimate for the spatial gradient of the heat kernel of the form

$$
\left|\nabla_{x} p_{t}(x, y)\right| \leq \frac{1}{t^{\frac{1}{2}}} \frac{1}{g(t)} \exp \left(-\frac{d^{2}(x, y)}{2 D t}\right), \quad x, y \in \mathcal{M}, t>0 .
$$

However, while heat kernel estimates have been extensively studied, much less is known about gradient estimates of this form. Such estimates are known to be equivalent in a rather general setting to the $L^{p}$-boundedness of the Riesz transform on $\mathcal{M}$. More precisely, for any non-compact manifold $\mathcal{M}$ whose heat kernel satisfies Gaussian estimates from above and below, one can show that the gradient of the heat kernel satisfies the $L^{p}-L^{p}$-estimate

$$
\left\|\nabla e^{\Delta t} u_{0}\right\|_{L^{p}} \leq C \frac{\left\|u_{0}\right\|_{L^{p}}}{t^{\frac{1}{2}}}
$$

for $p$ ranging in an open interval over 2, if and only if the Riesz transform is $L^{p}$ bounded in the same interval of $p$ 's (see [1]).

Some gradient estimates have been proved via probabilistic methods (see for example $[24,28,9,27,16,31,32]$ ). However, unless one assumes nonnegativity of the Ricci curvature, all these methods are limited so far to small times, more precisely they yield the crucial factor $\frac{1}{\sqrt{t}}$ only for $t$ near 0 . More precise results are available under additional assumptions on the modulus of continuity of the gradient of the heat kernel (see [20]) or for manifolds $\mathcal{M}$ that are Galois coverings of compact manifolds with nilpotent transformation group $G$ (see [13]). $L^{p}$-boundedness of the gradient of the heat kernel also holds on Cartan-Hadamard manifold whose Laplace operator is strictly positive, which satisfy some further bounds on the curvature tensor and its two first derivatives (see [25]). Nevertheless, the question of finding weaker purely geometric conditions for $L^{p}-L^{q}$ estimates to hold is so far completely open.

In the following we shall list a few sufficient conditions for the gradient estimates to hold globally in time.

First, in analogy with the heat kernel estimates, we shall introduces some weighted integrals which will provide us Gaussian estimates for the derivatives of the heat kernel.

Notation. We shall keep the following convention: $\nabla^{n}$ denotes $\Delta^{\frac{n}{2}}$ when $n$ is even and $\nabla \Delta^{\frac{n-1}{n}}$ when $n$ is odd.

Fix any $D>2$ and define

$$
E_{D, n}(x, t):=\int_{\mathcal{M}} \nabla_{y}^{n} p_{t}(x, z)^{2} \exp \left(\frac{d^{2}(x, z)}{D t}\right) d \mu(z),
$$

where $n \in \mathbb{N}$ and $x \in \mathcal{M}, t>0$ are arbitrary. Thus in particular $E_{D, 0} \equiv E_{D}$.

Fix $T \in] 0,+\infty]$. Given an integrable positive function $f(t)$ associate to it in $] 0, T[$ the sequence of integrals

$$
f_{0}=f, \quad f_{n}(t)=\int_{0}^{t} f_{n-1}(\tau) d \tau, \quad n \geq 1
$$


or, equivalently,

$$
f_{n}(t)=\int_{0}^{t} \frac{(t-\tau)^{n-1}}{(n-1) !} f(\tau) d \tau
$$

Theorem 2.6 ([20]). Suppose that for some $x \in \mathcal{M}$, for some constant $C$ and for every $t \in] 0, T[$

$$
E_{D, 0}(x, t) \leq \frac{C}{f(t)},
$$

where $f(t)$ is positive and integrable on $] 0, T[$. Then for every $n \geq 1$ and for every $t \in] 0, T[$

$$
E_{D, n}(x, t) \leq \frac{C c^{-n}}{f_{n}(t)}
$$

where

$$
c=\frac{D-2}{\frac{D}{2}+8} .
$$

The temporal derivatives of the heat kernel can be easily estimated through the integrals $\left\{E_{D, n}\right\}$ (see $[18,20]$ ), but it seems like it is not possible to obtain estimates for spatial derivatives of the heat kernel with the same degree of generality as for temporal derivatives. Indeed, it is well known that spatial derivatives of a solution to a parabolic or elliptic equation cannot be only controlled through the $L^{\infty}$ norm of the coefficients, further informations on the continuity modulus of the solution are needed.

However, if we know a priori that $\nabla_{x} p_{t}(x, y)$ and $\nabla_{y} p_{t}(x, y)$ are finitely proportional, we can easily estimate the gradient of the heat kernel just by deriving the semigroup identity:

Theorem 2.7 ([20]). Suppose that for some point $x \in \mathcal{M}$ and for all $y \in \mathcal{M}, t>$ 0 the following inequality holds:

$$
\left|\nabla_{x} p_{t}(x, y)\right| \leq C\left|\nabla_{y} p_{t}(x, y)\right|,
$$

then for every $y \in \mathcal{M}$ and for every $t>0$ we have

$$
\left|\nabla_{x} p_{t}(x, y)\right| \leq C \sqrt{E_{D, 1}\left(x, \frac{t}{2}\right) E_{D, 0}\left(y, \frac{t}{2}\right)} \exp \left(-\frac{d^{2}(x, y)}{2 D t}\right) .
$$

Proof. Deriving the semigroup identity

$$
p_{t}(x, y)=\int_{\mathcal{M}} p_{\frac{t}{2}}(x, z) p_{\frac{t}{2}}(y, z) d \mu(z)
$$

we obtain

$$
\nabla_{x} p_{t}(x, y)=\int_{\mathcal{M}} \nabla_{x} p_{\frac{t}{2}}(x, z) p_{\frac{t}{2}}(y, z) d \mu(z)
$$


Let $x, y, z$ be three points on $\mathcal{M}$; denote $\alpha=d(y, z), \beta=d(x, z)$ and $\gamma=$ $d(x, y)$. By triangular inequality $\alpha^{2}+\beta^{2} \geq \frac{1}{2} \gamma^{2}$. Then we have

$$
\begin{aligned}
\left|\nabla_{x} p_{t}(x, y)\right| & \leq \int_{\mathcal{M}}\left|\nabla_{x} p_{\frac{t}{2}}(x, z)\right| p_{\frac{t}{2}}(y, z) d \mu(z) \\
& \leq C \int_{\mathcal{M}}\left|\nabla_{y} p_{\frac{t}{2}}(x, z)\right| p_{\frac{t}{2}}(y, z) d \mu(z) \\
& \leq C \int_{\mathcal{M}} e^{\frac{\beta^{2}}{D t}}\left|\nabla_{y} p_{\frac{t}{2}}(x, z)\right| e^{\frac{\alpha^{2}}{D t}} p_{\frac{t}{2}}(y, z) e^{\frac{-\gamma^{2}}{2 D t}} d \mu(z) \\
& \leq C \sqrt{E_{D, 1}\left(x, \frac{t}{2}\right) E_{D, 0}\left(y, \frac{t}{2}\right)} \exp \left(-\frac{d^{2}(x, y)}{2 D t}\right),
\end{aligned}
$$

which is what we wanted to prove.

Remark. In particular, when $f(t)=t^{\frac{m}{2}}$, the previous estimate implies that

$$
\left|\nabla_{x} p_{t}(x, y)\right| \leq \frac{C}{t^{\frac{1}{2}+\frac{m}{2}}} \exp \left(-\frac{d^{2}(x, y)}{2 D t}\right) .
$$

From the upper bound of the previous theorem one can easily get the $L^{\infty}-L^{1}$-estimate. For the $L^{p}$-boundedness of the gradient, in contrast with the case of the kernel where it follows automatically from the maximum principle, we will need some more conditions on the manifold. The following result of Dungey gives us a sufficient condition for it to hold:

Theorem 2.8 (Dungey [14]). Let $\mathcal{M}$ be a connected, complete Riemannian manifold. Assume that the following properties hold on $\mathcal{M}$ :

- Volume doubling property: there exists $c>0$ such that

$$
V(x, 2 r) \leq c V(x, r)
$$

- $\quad$ The Gaussian heat kernel upper bound

$$
p_{t}(x, y) \leq c_{D} V(y, \sqrt{t})^{-1} \exp \left(-\frac{d^{2}(x, y)}{2 D t}\right) .
$$

Then for any $p \in[1, \infty]$ the following conditions are equivalent:

(i) There exists $c>0$ such that

$$
\left|\nabla_{x} p_{t}(x, y)\right| \leq c t^{-\frac{1}{2}} V(y, \sqrt{t})^{-1} \exp \left(-\frac{d^{2}(x, y)}{2 D t}\right)
$$

for all $t>0, x, y \in \mathcal{M}$;

(ii) There is $c>0$ such that

$$
\left|\nabla_{x} p_{t}(x, y)\right| \leq c t^{-\frac{1}{2}} V(y, \sqrt{t})^{-1}
$$

for all $t>0, x, y \in \mathcal{M}$;

(iii) There exists $c_{p}>0$ such that

$$
\left\|\nabla_{x} p_{t}(x, \cdot)\right\|_{L^{p}} \leq c t^{-\frac{1}{2}} V(x, \sqrt{t})^{-\left(1-\frac{1}{p}\right)}
$$

for all $t>0$ and $x \in \mathcal{M}$. 
Summing up, if the volumes of the balls on a manifold $\mathcal{M}$ satisfying the Faber-Krahn inequality (25) grow as $t^{\frac{m}{2}}$, we have that both (30) and (32) hold, and this yields a sufficient condition for the estimates in the second part of Theorem 1.2 to hold.

We recall that estimates (9-10) are also valid on any manifold $\mathcal{M}$ which is a Galois covering of a compact manifold, with nilpotent deck transformation group. This means that a finitely generated discrete group $G$ acts smoothly, properly and freely on the manifold $\mathcal{M}$ (non-compact, complete and connected) by isometries, such that the orbit space $\mathcal{M}^{\prime}=\mathcal{M} / G$ is a compact manifold.

For such manifolds polynomial volume growth is a consequence of the polynomial growth of the transformation group (see [6]), while a bound for the kernel of the form (32) is well known (see for example [30]).

In this setting, also gradient estimates are available, as proved by Dungey:

Theorem 2.9 (Dungey [13]). Assume $\mathcal{M}$ is a a Galois covering of a compact Riemannian manifold $\mathcal{M}^{\prime}$, with deck tranformation group $G$ of polynomial growth (e.g., a nilpotent group $G$ ). Then the following estimate holds:

$$
\left|\nabla_{x} p_{t}(x, y)\right| \leq c t^{-\frac{1}{2}} V(y, \sqrt{t})^{-1} \exp \left(-\frac{d^{2}(x, y)}{2 D t}\right) .
$$

\section{Proof of Theorem $\mathbf{1 . 2}$}

(i) As to the (5) let us first observe that - by interpolation - it is sufficient to prove the following estimates

$$
\begin{gathered}
\left\|e^{\Delta t} u_{0}\right\|_{L^{\infty}} \leq \frac{1}{g(t)}\left\|u_{0}\right\|_{L^{1}}, \\
\left\|e^{\Delta t} u_{0}\right\|_{L^{\infty}} \leq\left\|u_{0}\right\|_{L^{\infty}}, \\
\left\|e^{\Delta t} u_{0}\right\|_{L^{1}} \leq\left\|u_{0}\right\|_{L^{1}} .
\end{gathered}
$$

From the kernel estimate (4) we obtain immediately the estimate

$$
\begin{aligned}
|u(x, t)| & \leq \int_{\mathcal{M}} p_{t}(x, y)\left|u_{0}(y)\right| d \mu(y) \\
& \leq \frac{1}{g(t)} \int_{\mathcal{M}} e^{-\frac{d^{2}(x, y)}{2 D t}}\left|u_{0}(y)\right| d \mu(y) \\
& \leq \frac{1}{g(t)} \int_{\mathcal{M}}\left|u_{0}(y)\right| d \mu(y) \\
& =\frac{1}{g(t)}\left\|u_{0}\right\|_{L^{1}},
\end{aligned}
$$

so, taking the supremum with respect to $x \in \mathcal{M}$, we get the (37):

$$
\|u(\cdot, t)\|_{L^{\infty}} \leq \frac{1}{g(t)}\left\|u_{0}\right\|_{L^{1}} .
$$


As to the second estimate, we can exploit the maximum principle (15), from which easily follows that

$$
|u(x, t)| \leq\left\|u_{0}\right\|_{L^{\infty}} \sup _{x \in \mathcal{M}} \int_{\mathcal{M}} p_{t}(x, y) d \mu(y) \leq\left\|u_{0}\right\|_{L^{\infty}} .
$$

Finally let us show the $L^{1}-L^{1}$ estimate:

$$
\begin{aligned}
\|u(\cdot, t)\|_{L^{1}} & \leq \int_{\mathcal{M}} \int_{\mathcal{M}} p_{t}(x, y)\left|u_{0}(y)\right| d \mu(y) d \mu(x) \\
& =\int_{\mathcal{M}} \int_{\mathcal{M}} p_{t}(x, y)\left|u_{0}(y)\right| d \mu(x) d \mu(y) \\
& =\int_{\mathcal{M}}\left|u_{0}(y)\right|\left(\int_{\mathcal{M}} p_{t}(y, x) d \mu(x)\right) d \mu(y) \\
& \leq \int_{\mathcal{M}}\left|u_{0}(y)\right| d \mu(y)=\left\|u_{0}\right\|_{L^{1}},
\end{aligned}
$$

where we used Tonelli's theorem and then the symmetry of the heat kernel, and finally we applied the maximum principle (15). The gradient estimate follows the same way, since we know from Sect. 2.2 that under our hypotheses (35) is equivalent to (6).

(ii) It is trivial once we notice that by the theory in Sects. 2.1 and 2.2, the hypoteses of point (i) hold with $g(t)=C t^{\frac{m}{2}}$.

\section{Proof of Theorem 1.3}

(i) Let us consider the integral equation associated to the Cauchy problem (3): let

$$
\mathscr{F} u(t):=e^{\Delta t} u_{0}+\int_{0}^{t} e^{\Delta(t-\tau)}[A(u)(\nabla u, \nabla u)(\tau)] d \tau .
$$

The idea is to use the contraction principle to show that for $T\left(u_{0}\right)$ small enough a fixed point for the integral operator $\mathscr{F}$ exists in $\mathcal{E}_{p}^{T}$ for all $T<T\left(u_{0}\right)$.

First let us show that $\mathscr{F}$ maps $\mathcal{E}_{p}^{T}$ into itself for $T$ small. Using the bound (11) and the estimate (9) in the case $L^{\infty}-L^{\frac{p}{2}}$, we can deduce that

$$
\begin{aligned}
\left\|\mathscr{F} u(t)-e^{\Delta t} u_{0}\right\|_{L^{\infty}} & \leq \int_{0}^{t}\left\|e^{\Delta(t-\tau)} A(u)(\nabla u, \nabla u)\right\|_{L^{\infty}} d \tau \\
& \leq \int_{0}^{t} \frac{C}{(t-\tau)^{\frac{m}{p}}}\|A(u)(\nabla u, \nabla u)(\tau)\|_{L^{\frac{p}{2}}} d \tau \\
& \leq \int_{0}^{t} \frac{C}{(t-\tau)^{\frac{m}{p}}}\left\||\nabla u(\tau)|^{2}\right\|_{L^{\frac{p}{2}}} d \tau \\
& =\int_{0}^{t} \frac{C}{(t-\tau)^{\frac{m}{p}}}\|\nabla u(\tau)\|_{L^{p}}^{2} d \tau
\end{aligned}
$$




$$
\leq C M^{2} \int_{0}^{T} \frac{d \tau}{\tau^{\frac{m}{p}}},
$$

and the last integral is finite whenever $p>m$.

Similarly, using the estimate (10) in the case $L^{p}-L^{\frac{p}{2}}$, we obtain the bound

$$
\begin{aligned}
\left\|\nabla \mathscr{F} u(t)-\nabla e^{\Delta t} u_{0}\right\|_{L^{p}} & \leq \int_{0}^{t} \frac{C}{(t-\tau)^{\frac{1}{2}+\frac{m}{2 p}}}\|A(u)(\nabla u, \nabla u)(\tau)\|_{L^{\frac{p}{2}}} d \tau \\
& \leq C M^{2} \int_{0}^{T} \frac{d \tau}{\tau^{\frac{1}{2}+\frac{m}{2 p}}},
\end{aligned}
$$

and once again the last integral converges when $p>m$.

Now, for $T$ small, $\mathscr{F}$ is a contraction on $\mathcal{E}_{p}^{T}$ : indeed for $u$ and $v$ in $\mathcal{E}_{p}^{T}$, using (9), we have

$$
\begin{array}{rl}
\| \mathscr{F} & u(t)-\mathscr{F} v(t) \|_{L^{\infty}} \\
\leq & \int_{0}^{t}\left\|e^{\Delta(t-\tau)}[A(u)(\nabla u, \nabla u)(\tau)-A(v)(\nabla v, \nabla v)(\tau)]\right\|_{L^{\infty}} d \tau \\
\leq & \int_{0}^{t} \frac{C}{(t-\tau)^{\frac{m}{p}}}\|A(u)(\nabla u, \nabla u)(\tau)-A(v)(\nabla v, \nabla v)(\tau)\|_{L^{\frac{p}{2}}} d \tau \\
\leq & \int_{0}^{t} \frac{C}{(t-\tau)^{\frac{m}{p}}}\left[\|\nabla u(\tau)\|_{L^{p}}^{2}\|u-v\|_{L^{\infty}}\right. \\
& \left.+\|\nabla u(\tau)-\nabla v(\tau)\|_{L^{p}}\left(\|\nabla u(\tau)\|_{L^{p}}+\|\nabla v(\tau)\|_{L^{p}}\right)\right] d \tau \\
\leq & C\left(M+M^{2}\right) \int_{0}^{T} \frac{d \tau}{\tau^{\frac{m}{p}}} \cdot d(u, v),
\end{array}
$$

and analogously thanks to (10) we can estimate

$$
\begin{aligned}
& \|\nabla \mathscr{F} u(t)-\nabla \mathscr{F} v(t)\|_{L^{p}} \\
& \quad \leq \int_{0}^{t}\left\|\nabla e^{\Delta(t-\tau)}[A(u)(\nabla u, \nabla u)(\tau)-A(v)(\nabla v, \nabla v)(\tau)]\right\|_{L^{p}} d \tau \\
& \quad \leq \int_{0}^{t} \frac{C}{(t-\tau)^{\frac{1}{2}+\frac{m}{2 p}}}\|A(u)(\nabla u, \nabla u)(\tau)-A(v)(\nabla v, \nabla v)(\tau)\|_{L^{\frac{p}{2}}} d \tau \\
& \quad \leq C\left(M+M^{2}\right) \int_{0}^{T} \frac{d \tau}{\tau^{\frac{1}{2}+\frac{m}{2 p}}} \cdot d(u, v) .
\end{aligned}
$$

As a consequence, from the contraction principle follows that, for $T$ small enough the operator $\mathscr{F}$ has a unique fixed point in $\mathcal{E}_{p}^{T}$. Since $A(u)(\nabla u, \nabla u) \in C\left(\left[0, T\left[; L^{p / 2}\right)\right.\right.$, we can differentiate $u$ and see that it satisfies (3) in a distributional sense.

The blow-up alternative can be deduced in a classical way.

(ii) To show that the solution previously obtained is global, it is sufficient to obtain an a priori estimate for the quantity $\|u(t)\|_{\mathcal{F}_{p}}=\|\nabla u(t)\|_{L^{p}}+$ 
$\|u(t)\|_{L^{\infty}}$. Using (11), (9) and (10), we have

$$
\|\nabla u(t)\|_{L^{p}} \leq C\left\{\frac{\left\|\nabla u_{0}\right\|_{L^{m}}}{t^{\frac{m}{2}\left(\frac{1}{m}-\frac{1}{p}\right)}}+\int_{0}^{t} \frac{\|\nabla u(\tau)\|_{L^{p}}^{2}}{(t-\tau)^{\frac{1}{2}+\frac{m}{2 p}}} d \tau\right\} .
$$

Let us define

$$
M_{p}(T):=\sup _{t \in[0, T]}\left\{t^{\frac{m}{2}\left(\frac{1}{m}-\frac{1}{p}\right)}\|\nabla u(t)\|_{L^{p}}\right\} .
$$

We have then for $t \in[0, T]$

$$
\begin{aligned}
& t^{\frac{m}{2}\left(\frac{1}{m}-\frac{1}{p}\right)}\|\nabla u(t)\|_{L^{p}} \\
& \quad \leq C\left\{\left\|\nabla u_{0}\right\|_{L^{m}}+M_{p}^{2}(T) \int_{0}^{t} \frac{t^{\frac{m}{2}\left(\frac{1}{m}-\frac{1}{p}\right)}}{(t-\tau)^{\frac{1}{2}+\frac{m}{2 p}} \tau^{m\left(\frac{1}{m}-\frac{1}{p}\right)}} d \tau\right\} \\
& \quad=C\left\{\left\|\nabla u_{0}\right\|_{L^{m}}+M_{p}^{2}(T) \int_{0}^{1} \frac{d y}{(1-y)^{\frac{1}{2}+\frac{m}{2 p}} y^{1-\frac{m}{p}}}\right\} \\
& \quad \leq C\left\|\nabla u_{0}\right\|_{L^{m}}+\bar{C} M_{p}^{2}(T),
\end{aligned}
$$

where

$$
\bar{C}=C \int_{0}^{1} \frac{d y}{y^{1-\frac{m}{p}}(1-y)^{\frac{1}{2}+\frac{m}{2 p}}} .
$$

Taking the supremum in $t$ in the left hand side member we obtain the inequality

$$
\bar{C} M_{p}^{2}(T)-M_{p}(T)+C\left\|\nabla u_{0}\right\|_{L^{m}} \geq 0,
$$

true for every $T \in] 0, T\left(u_{0}\right)[$.

The polynomial $\bar{C} M_{p}^{2}(T)-M_{p}(T)+C\left\|\nabla u_{0}\right\|_{L^{m}}$ has two distinct real roots when $\left\|\nabla u_{0}\right\|_{L^{m}}<\frac{1}{4 C \bar{C}}=: \epsilon$. Suppose that this is the case and denote $M_{p}^{\infty}$ the smallest root. Since the map $T \mapsto M_{p}(T)$ is continuous, and $M_{p}(0)=0$, we have immediately $M_{p}(T) \leq M_{p}^{\infty}$ for all $T$, and so

$$
\|\nabla u(t)\|_{L^{p}} \leq \frac{M_{p}^{\infty}}{t^{\frac{1}{2}-\frac{m}{2 p}}} .
$$

Using the estimate we obtained for $\|\nabla u\|_{L^{p}}$, we get

$$
\begin{aligned}
\|u(t)\|_{L^{\infty}} & =\|\mathscr{F} u(t)\|_{L^{\infty}} \\
& \leq\left\|u_{0}\right\|_{L^{\infty}}+\int_{0}^{t} \frac{C}{(t-\tau)^{\frac{m}{p}}}\|\nabla u(\tau)\|_{L^{p}}^{2} d \tau \\
& \leq\left\|u_{0}\right\|_{L^{\infty}}+C\left(M_{p}^{\infty}\right)^{2} \int_{0}^{t} \frac{d \tau}{(t-\tau)^{\frac{m}{p}} \tau^{1-\frac{m}{p}}} \\
& =\left\|u_{0}\right\|_{L^{\infty}}+C\left(M_{p}^{\infty}\right)^{2} \int_{0}^{1} \frac{d y}{(1-y)^{\frac{m}{p}} y^{1-\frac{m}{p}}} .
\end{aligned}
$$


Summing up the estimates that we found we obtain an a priori estimate for $\|u(t)\|_{\mathcal{F}_{p}}$. Such an estimate makes us sure that $u(t)$ cannot blow-up in finite time in $\mathcal{F}_{p}$, so that it is a global solution to (3).

Moreover let us observe that since $u \in C\left(\left[0,+\infty\left[; \mathcal{F}_{p}\right)\right.\right.$, it is indeed a solution to the homotopy problem.

\section{Acknowledgments}

I wish to express all my gratitude to Prof. Piero D'Ancona for many useful discussions and for guiding me throughout the writing process.

\section{References}

[1] Auscher, P., Coulhon, T., Duong, X.T., Hofmann, S.: Riesz transform on manifolds and heat kernel regularity. Ann. Sci. École Norm. Sup. (4) 37(6), 911957 (2004)

[2] Carlen, E.A., Kusuoka, S., Stroock, D.W.: Upper bounds for symmetric Markov transition functions. Ann. Inst. H. Poincaré Probab. Statist. 23(2 suppl), 245$287(1987)$

[3] Carron, G.: Inégalités isopérimétriques de Faber-Krahn et conséquences. Actes de la Table Ronde de Géométrie Différentielle (Luminy, 1992), 205-232, Sémin. Congr., 1, Soc. Math. France, Paris (1996)

[4] Chang, K.-C., Ding, W.Y., Ye, R.: Finite-time blow-up of the heat flow of harmonic maps from surfaces. J. Differential Geom. 36(2), 507-515 (1992)

[5] Chavel, I.: Eigenvalues in Riemannian geometry Pure and Applied Mathematics 115. Academic Press, Inc, Orlando (1984)

[6] Chavel, I.: Isoperimetric inequalities. Differential geometric and analytic perspectives. Cambridge Tracts in Mathematics, 145. Cambridge University Press, Cambridge (2001)

[7] Coron, J.M.: Nonuniqueness for the heat flow of harmonic maps. Ann. Inst. H. Poincaré Anal. Non Linéaire 7(4), 335-344 (1990)

[8] Coulhon, T.: Ultracontractivity and Nash type inequalities. J. Funct. Anal. 141(2), 510-539 (1996)

[9] Cranston, M.: Gradient estimates on manifolds using coupling. J. Funct. Anal. 99(1), 110-124 (1991)

[10] Davies, E.B.: Explicit constants for Gaussian upper bounds on heat kernels. Amer. J. Math. 109(2), 319-333 (1987)

[11] Davies, E.B.: Gaussian upper bounds for the heat kernels of some second-order operators on Riemannian manifolds. J. Funct. Anal. 80(1), 16-32 (1988) 
[12] Davies, E.B.: Heat kernels and spectral theory. Cambridge Tracts in Mathematics, 92. Cambridge University Press, Cambridge (1989)

[13] Dungey, N.: Heat kernel estimates and Riesz transforms on some Riemannian covering manifolds. Math. Z. 247(4), 765-794 (2004)

[14] Dungey N.: Some remarks on gradient estimates for heat kernels. (English summary) Abstr. Appl. Anal. Art. ID 73020, 10 (2006)

[15] Eells, J. Jr., Sampson, J.H.: Harmonic mappings of Riemannian manifolds. Amer. J. Math 86, 109-160 (1964)

[16] Elworthy, K.D., Li, X.-M.: Formulae for the derivatives of heat semigroups. J. Funct. Anal. 125(1), 252-286 (1994)

[17] Grigor'yan, A.: On the fundamental solution of the heat equation on an arbitrary Riemannian manifold. (Russian) Mat. Zametki 41 (1987), (5) 687-692, 765. English translation: Math. Notes 41 (1987), no. 5-6, 386-389

[18] Grigor'yan, A.: Gaussian upper bounds for the heat kernel and for its derivatives on a Riemannian manifold. Classical and modern potential theory and applications (Chateau de Bonas 1993). NATO Adv. Sci. Inst. Ser. C Math. Phys. Sci. pp. 237-252. Kluwer Academic Publishers, Dodrecht (1994)

[19] Grigor'yan, A.: Heat kernel upper bounds on a complete non-compact manifold. Rev. Mat. Iberoamericana 10(2), 395-452 (1994)

[20] Grigor'yan, A.: Upper bounds of derivatives of the heat kernel on an arbitrary complete manifold. J. Funct. Anal. 127(2), 363-389 (1995)

[21] Grigor'yan, A.: Gaussian upper bounds for the heat kernel on arbitrary manifolds. J. Differential Geom. 35(1), 33-52 (1997)

[22] Grigor'yan, A.: Estimates of heat kernels on Riemannian manifolds. Spectral theory and geometry (Edinburgh, 1998), 140-225. London Math. Soc. Lecture Note Ser., 273, Cambridge University Press, Cambridge (1999)

[23] Grigor'yan, A.: Heat kernels on weighted manifolds and applications. The ubiquitous heat kernel. Contemp. Math. 398. pp. 93-191. Math. Soc. Providence, RI (2006)

[24] Li, P., Yau, S.-T.: On the parabolic kernel of the Schrödinger operator. Acta Math. 156(3-4), 153-201 (1986)

[25] Lohoué, N.: Comparaison des champs de vecteurs et des puissances du laplacien sur une varit riemannienne courbure non positive. J. Funct. Anal. 61(2), 164201 (1985)

[26] Nash, J.: The imbedding problem for Riemannian manifolds. Ann. Math. (2) 63(1), 20-63 (1956)

[27] Picard, J.: Gradient estimates for some diffusion semigroups. Probab. Theory Rel. Fields 122(4), 593-612 (2002) 
[28] Qian, Z.M.: Gradient estimates and heat kernel estimate. Proc. Roy. Soc. Edinburgh Sect. A 125(5), 975-990 (1995)

[29] Soyeur, A.: A global existence result for the heat flow of harmonic maps. Comm. Partial Differ. Equ. 15(2), 237-244 (1990)

[30] Stein, E.M.: Harmonic analysis: real-variable methods, orthogonality, and oscillatory integrals. Princeton Mathematical Series, 43. Monographs in Harmonic Analysis, III. Princeton University Press, Princeton NJ (1993)

[31] Stroock, D.W., Turetsky, J.: Upper bounds on derivatives of the logarithm of the heat kernel. Comm. Anal. Geom. 6(4), 669-685 (1998)

[32] Thalmaier, A., Wang, F.-T.: Gradient estimates for harmonic functions on regular domains in Riemannian manifolds. J. Funct. Anal. 155(1), 109-124 (1998)

[33] Ušakov V.I.: Stabilization of solutions of the third mixed problem for a second-order parabolic equation in a noncylindrical domain. (Russian) Mat. Sb. (N.S.) 111(153) (1980), (1), 95-115, 159. English translation: Math. USSR-Sb. 39 (1981), 87-105

[34] Varopoulos, N.T.: Hardy-Littlewood theory for semigroups. J. Funct. Anal. 63(2), 240-260 (1985)

\section{Paniccia}

Mathematisches Institut,

Universität Bonn,

Endenicher Allee 60,

53115 Bonn,

Germany

e-mail: paniccia@math.uni-bonn.de

Received: 24 April 2010.

Accepted: 20 December 2010. 